\title{
Near-Infrared Spatial Self-Phase Modulation in Ultrathin Niobium Carbide Nanosheets
}

\author{
Si Xiao ${ }^{1}$, Yi-lin He ${ }^{1}$, Yu-lan Dong ${ }^{2}$, Yi-duo Wang ${ }^{1}$, Li Zhou ${ }^{1}$, Xue-jun Zhang ${ }^{1}$, \\ Ying-wei Wang ${ }^{1 *}$ and Jun $\mathrm{He}^{1 *}$ \\ ${ }^{1}$ Hunan Key Laboratory of Nanophotonics and Devices, School of Physics and Electronics, Central South University, \\ Changsha, China, ${ }^{2}$ Key Laboratory of Hunan Province for Statistical Learning and Intelligent Computation, School of \\ Mathematics and Statistics, Hunan University of Technology and Business, Changsha, China
}

OPEN ACCESS

Edited by:

Jun Wang,

University of Electronic Science and Technology of China, China

Reviewed by:

Chujun Zhao,

Hunan University, China

Leiming Wu,

Guangdong University of

Technology, China

${ }^{*}$ Correspondence:

Ying-wei Wang

wyw1988@csu.edu.cn Jun $\mathrm{He}$

junhe@csu.edu.cn

Specialty section: This article was submitted to

Optics and Photonics,

a section of the journal

Frontiers in Physics

Received: 02 March 2021 Accepted: 06 April 2021

Published: 07 May 2021

Citation:

Xiao S, He Y-I, Dong Y-I, Wang Y-d, Zhou L, Zhang $X-j$, Wang $Y-W$ and He J (2021) Near-Infrared Spatial Self-Phase Modulation in Ultrathin Niobium Carbide Nanosheets.

Front. Phys. 9:674820

doi: 10.3389/fphy.2021.674820
Spatial self-phase modulation (SSPM) as a purely coherent non-linear optical effect (also known as Kerr effect) can support strong broadband phase modulation, which is essential for all-optical applications. Besides this, the increasing use of two-dimensional (2D) materials opens up new prospects in this field of research. In this work, we report a broadband SSPM response from 2D transition metal carbonitrides (MXenes) and $\mathrm{Nb}_{2} \mathrm{C}$, arising in the near-infrared $(1,550 \mathrm{~nm})$ range. Based on the SSPM measurements of few-layer $\mathrm{Nb}_{2} \mathrm{C}$ nanosheets, the third-order non-linear optical parameters of $\mathrm{Nb}_{2} \mathrm{C}$, including the non-linear refractive index $n_{2}$ and susceptibility $\chi^{(3)}$, were determined at 400, 800, 1,300, and 1,550 nm. Moreover, the physics mechanism of the dynamic formation process of SSPM diffraction rings was exploited. The formation time of SSPM diffraction rings can be divided into two typical parts which correspond to the polarization and reorientation of $2 \mathrm{D} \mathrm{Nb}{ }_{2} \mathrm{C}$ nanosheets. As a proof of concept, we demonstrate the nonreciprocal light propagation at wavelengths of 1,300 and $1,550 \mathrm{~nm}$ by constructing an $\mathrm{Nb}_{2} \mathrm{C} /$ water hybrid structure. Our results reveal strong optical phase modulation of $\mathrm{Nb}_{2} \mathrm{C}$ in the infrared region, thus showing the great potential of MXene materials for use in passive photonic devices.

Keywords: spatial self-phase modulation, niobium carbide $\left(\mathrm{Nb}_{2} \mathrm{C}\right)$, non-linear optics, $2 \mathrm{D}$ materials, infrared rage

\section{INTRODUCTION}

Spatial self-phase modulation (SSPM) is one of the typical third-order optical processes $[1,2]$. It provides a way to measure the non-linear refractive index and third-order nonlinear susceptibility of a medium. Owing to their fascinating non-linear photonics applications $[3,4]$, tremendous efforts have been made to explore the non-linear optical properties of 2D materials including graphene [5, 6], transition metal chalcogenides (TMDCs) [7], black phosphorus [8-10], and MXenes [11, 12]. Previous studies report on SSPM in various 2D materials such as graphene [13], $\mathrm{MoS}_{2}$ [14, 15], $\mathrm{MoTe}_{2}$ [16], tellurium [17], $\mathrm{NbSe}_{2}$ [18], and so on [19-26]. Over the past years, large-scale production of 2D materials has enabled their application in all-optical switches, all-optical modulators, and passive photonic diodes [27]. For example, excitation of the SSPM in $\mathrm{MoS}_{2}$ allows one to achieve the two-color and high-contrast all-optical switching with a full contrast ratio, $\mathrm{I}_{\text {weak }}: \mathrm{I}_{\text {strong }}$ $=1: 60$ [28]. Furthermore, non-reciprocal light propagation was observed in a solid-state graphdiyne/ $\mathrm{SnS}_{2}$-based photonic diode [27]. Thereafter, an all-optical information carrier and conversion system was designed to transmit/convert information $[18,19]$. To gain insight into 
the underlying mechanism of SSPM, numerous theoretical investigations have been carried out to promote experimental research in this field of study [29, 30].

Intrinsically, 2D materials are attracting increasing attention due to their unique optical modulation in a visible region; however, experimental evidence of the infrared light response of the SSPM in 2D materials has been rarely reported so far [31]. In particular, the SSPM patterns of black phosphorus were successfully acquired by our group at a wavelength of 1,160 nm [32]. Very recently, the use of SSPM technique allowed one to measure the third-order non-linear susceptibility of $\mathrm{Ti}_{3} \mathrm{C}_{2} \mathrm{~T}_{\mathrm{X}}$ MXene within a visible-to-near-infrared (Vis-NIR) range (400$1,064 \mathrm{~nm}$ ) [33]. The near-infrared region, especially presented by the optical communication band, is essential for all-optical modulation applications of the SSPM as it ensures the low-loss characteristics of the optical fiber communications.

Belonging to a class of two-dimensional (2D) inorganic compounds such as transition-metal carbides, nitrides, and carbonitrides, MXenes share a general formula of $\mathrm{M}_{\mathrm{n}+1} \mathrm{X}_{\mathrm{n}}(n$ $=1-3$ ), where $\mathrm{M}$ is an early transition metal (e.g., $\mathrm{Ti}, \mathrm{Zr}$, $\mathrm{V}, \mathrm{Nb}, \mathrm{Ta}$, and $\mathrm{Mo}$ ), and $\mathrm{X}$ is a $\mathrm{C}$ or $\mathrm{N}$. As a new member of the MXene family, $\mathrm{Nb}_{2} \mathrm{C}$ has attracted great attention due to its excellent performance in the fields of energy storage [34], electrochemical catalytic [35], and biomedicine [36]. Being semimetals with fascinating electrical properties, few-layer $\mathrm{Nb}_{2} \mathrm{C}$ nanosheets exhibit a narrow optical bandgap which favors the infrared optical response. Moreover, their superior photothermal conversion efficiency in the infrared region has even been proven in photothermal cancer therapy [36].

In this work, we investigate the near-infrared SSPM response of few-layer $\mathrm{Nb}_{2} \mathrm{C}$ nanosheets and expand SSPM response of $2 \mathrm{D}$ material to the communication band which cover $1,550 \mathrm{~nm}$ wavelength. The third-order non-linear susceptibility and the non-linear refractive index of $\mathrm{Nb}_{2} \mathrm{C}$ were measured within a broadband range from 400 to $1,550 \mathrm{~nm}$. Moreover, to understand the physics mechanism behind this, we exploited the dynamic formation process of SSPM diffraction rings. Based on the time of the rings formation, the dynamic formation process of SSPM diffraction rings can be divided into two typical time stages which correspond to the polarization and reorientation of $2 \mathrm{D}$ $\mathrm{Nb}_{2} \mathrm{C}$ nanosheets. Further, we demonstrate the proof-of-concept $\mathrm{Nb}_{2} \mathrm{C}$ non-reciprocal light propagation. During the exploitation of a passive photonic device, water was used instead of reverse saturable absorption materials $[17,27]$ to construct a $\mathrm{Nb}_{2} \mathrm{C} /$ water asymmetric hybrid structure. This enabled us to achieve the non-reciprocal light propagation in the near-infrared region by a facile way.

\section{EXPERIMENTAL}

\section{Synthesis of $\mathrm{Nb}_{2} \mathrm{C}$ Nanosheets}

The $\mathrm{Nb}_{2} \mathrm{AlC}$ was purchased from 11 Technology Company Limited, the size of which is about 400 mesh. Ten grams of the bulk $\mathrm{Nb}_{2} \mathrm{AlC}$ powder was added into $80 \mathrm{ml}$ of a $40 \% \mathrm{HF}$ solution (Macklin Incorporation). The suspension was then vigorously stirred for $48 \mathrm{~h}$ at $25^{\circ} \mathrm{C}$. After that, the $\mathrm{HF}$ solution was removed and the multilayer $\mathrm{Nb}_{2} \mathrm{C}$ was washed with water by centrifugation until the $\mathrm{pH}$ of water was about 5 . The tetrapropylammonium hydroxide (TPAOH) was then poured into $\mathrm{Nb}_{2} \mathrm{C}$ and stirred for $72 \mathrm{~h}$. Finally, the selected few-layer $\mathrm{Nb}_{2} \mathrm{C}$ nanosheets were collected by centrifugation.

\section{SSPM Measurements}

The experimental setup is shown in Figure 2A. The light source is a mode-locked Ti:sapphire regenerative amplifier system (Spectra-Physics, Spitfire ACE-35F-2KXP, Maitai SP, and Empower 30). Femtosecond laser pulse has a very short action time with materials, which cause a small thermal effect compared with continuous laser and long pulse laser (ns, ms). The transmitted light was captured by a charge coupled device (CCD) detector (LBP2-VIS2 was applied at wavelengths of 400, 800, and 1,300 $\mathrm{nm}$; and LBP2-HR-IR2 at 1,550 nm).

\section{Sample Characterization}

The scanning electron microscope (SEM) images were captured by field-emission Magellan 400 microscope (FEI Company). The transmission electron microscopy (TEM), high-angle annular dark field (HAADF), and energy dispersive X-ray spectroscopy (EDS) data were acquired on a JEM-3,100 (FEI Talos S-FEG) transmission electron microscope. The X-ray diffraction (XRD) patterns were recorded using a BRUKER D8 ADVANCE XRD system. The Ultraviolet-Visible-Near Infrared Rage (UV-VisNIR) absorption spectra from 200 to $1,600 \mathrm{~nm}$ of $\mathrm{Nb}_{2} \mathrm{C}$ were measured by a UV-vis spectrophotometer (Cary60, Agilent). Atomic Force Microscope (AFM) image was captured by CSPM 5,500.

\section{RESULTS AND DISCUSSION}

A typical two-step exfoliation strategy was employed to obtain $\mathrm{Nb}_{2} \mathrm{C}$ nanosheets (see Experimental section). Briefly, the $\mathrm{Nb}_{2}$ AlC MAX phase was etched in hydrofluoric (HF) acid solution to remove the $\mathrm{Al}$ atoms. Ultrasound-assisted $\mathrm{TPAOH}$ co-intercalated exfoliation was effective to reduce the lateral size and the thickness of the obtained $\mathrm{Nb}_{2} \mathrm{C}$ nanosheet [12]. Figure $1 \mathrm{~A}$ shows the SEM images of $\mathrm{Nb}_{2} \mathrm{AlC}$ powder. Before the $\mathrm{HF}$ etching, the MAX-phase $\mathrm{Nb}_{2} \mathrm{AlC}$ had the particle size of several or tens of micrometers along with a high interlayer bond strength. Inset in panel $\mathrm{A}$ is the zoom-in images of the single $\mathrm{Nb}_{2} \mathrm{AlC}$ particle which exhibits dense cross section. After the removal of $\mathrm{Al}$ atoms from the $\mathrm{Nb}_{2} \mathrm{AlC}$, the multi-layer $\mathrm{Nb}_{2} \mathrm{C}$ was formed like an accordion pulled open, as shown in Figure 1B. As can be seen in Figure 1C, the AFM measurement of present few-layer $\mathrm{Nb}_{2} \mathrm{C}$ was performed to characterize its thickness of about 3-4 nm. Figure 1d1 depicts the TEM images of $\mathrm{Nb}_{2} \mathrm{C}$ nanosheets, revealing their transparent morphology and ultrathin structure. As shown in Figures 1d2-d4, HAADF and EDS mapping results of $\mathrm{Nb}_{2} \mathrm{C}$ nanosheets with clear boundaries confirm the coexistence of $\mathrm{Nb}$ and $\mathrm{C}$ elements, which provides evidence for the elemental composition of as-prepared $2 \mathrm{D} \mathrm{Nb} \mathrm{N}_{2} \mathrm{C}$ nanosheets. Figure 1E displays the XRD patterns of samples. Two sharp peaks $\theta=12.8$ and $38.8^{\circ}$ are observed in the case of $\mathrm{Nb}_{2} \mathrm{AlC}$, which is in good agreement with JCDS PDF\#300033 for $\mathrm{Nb}_{2} \mathrm{AlC}$. The disappearance of the peak at $\theta=38.8^{\circ}$ indicates that the $\mathrm{Al}$ atoms have been successfully removed after etching in HF solution. The downshift of the peak at $\theta=12.8^{\circ}$ 


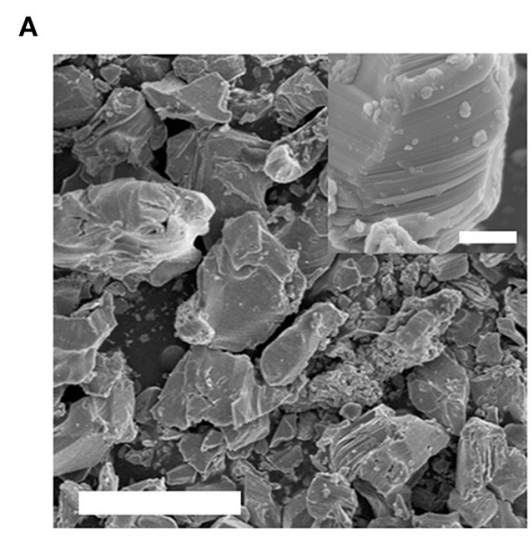

D

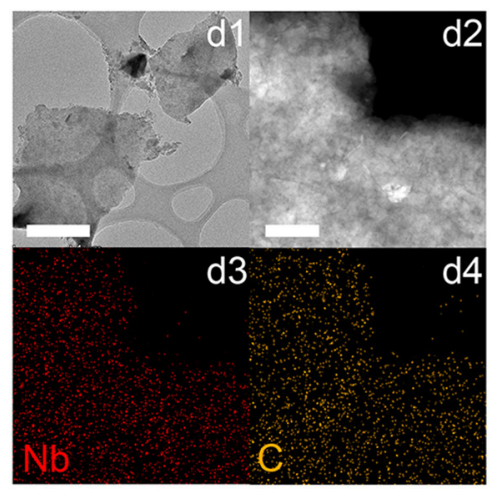

B

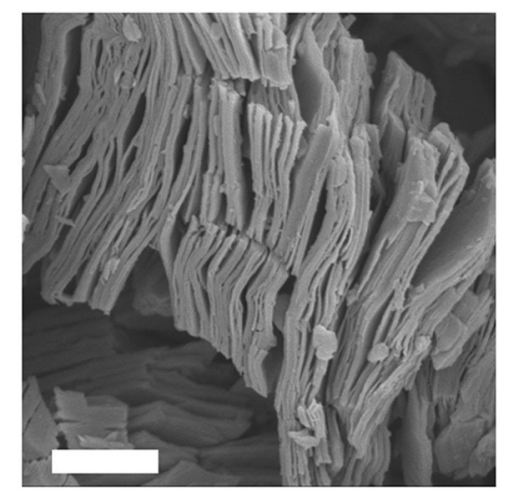

E

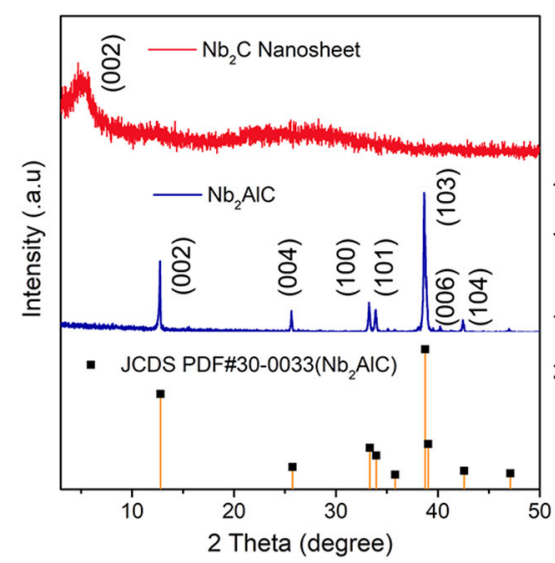

C

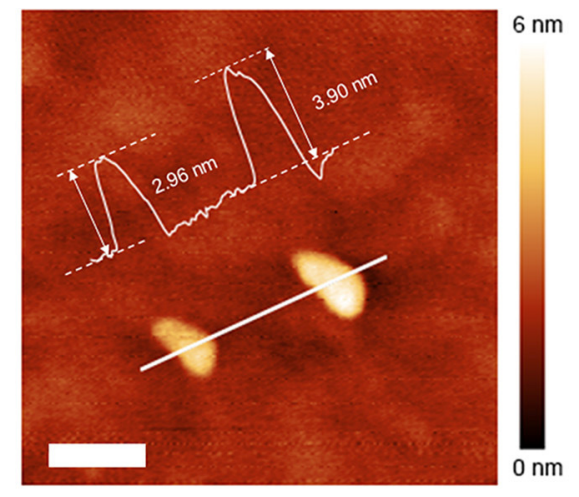

F

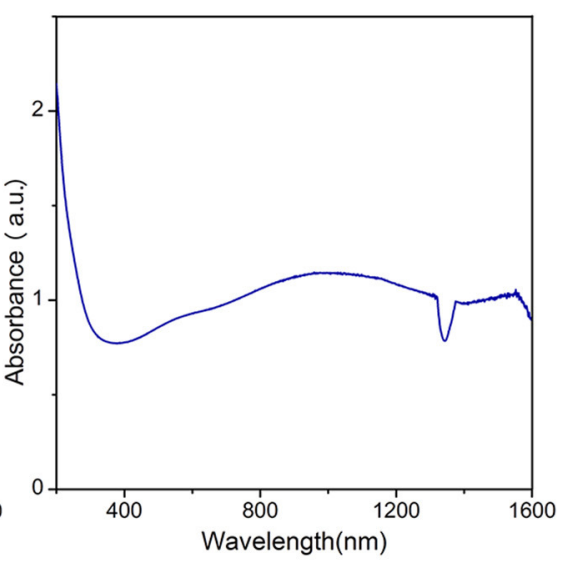

FIGURE 1 | (A) SEM images of $\mathrm{Nb}_{2} \mathrm{AIC}$ powder. Scale bars are 10 and $1 \mu \mathrm{m}$ in (A) and inset. (B) SEM image of multilayer $\mathrm{Nb}{ }_{2} \mathrm{C}$. Scale bar is $1 \mu \mathrm{m}$. (C) AFM image of $\mathrm{Nb}_{2} \mathrm{C}$. Scale bar is $200 \mathrm{~nm}$. (D,d1) TEM image, and (d2) HAADF image. (d3,d4) are EDS mapping of few-layer Nb ${ }_{2} \mathrm{C}$ nanosheets. Scale bars are $1 \mu \mathrm{m}$ and $200 \mathrm{~nm}$ in (d1,d2). (E) XRD patterns of $\mathrm{Nb}_{2} \mathrm{C}$ (upper) and $\mathrm{Nb}_{2} \mathrm{AIC}$ (lower). (F) UV-vis-NIR absorption spectra of $\mathrm{Nb}_{2} \mathrm{C}$.

and its broadening mean that MAX is converted into MXene. Figure $1 \mathrm{~F}$ shows the UV-Vis-NIR absorption spectra of $\mathrm{Nb}_{2} \mathrm{C}$ (To note, the gap may attribute to be an error from instruments.) The abruptly enhanced near-IR absorption suggests a strong light-matter interaction within the near-infrared range.

The SSPM measurements of $\mathrm{Nb}_{2} \mathrm{C}$ nanosheets were carried out at four different wavelengths including the light communication band at $1,550 \mathrm{~nm}$ (Figure 2). The solvent we use is N-methyl pyrrolidone (NMP) which possessed low volatility, good thermal, and chemical stability. To exclude the influence of solvents to the formation of diffraction rings, the control group was set in which only the NMP solvent was contained for SSPM experiments. There are no diffraction rings for NMP at 400, 800, 1,300, and 1,550 nm. Figure $2 \mathrm{~A}$ shows the experimental configuration used in the SSPM measurements. The typical SSPM diffraction patterns at different laser excitation wavelengths (from 400 to $1,550 \mathrm{~nm}$ ) were captured by a CCD detector, as shown in Figures 2B-E. The distinguishable diffraction rings are observed at a wavelength of $1,550 \mathrm{~nm}$, which indicates its potential versatility for optoelectronic applications within a range of a near-infrared telecom C band.
The quantitative information about the non-linear optical response of $\mathrm{Nb}_{2} \mathrm{C}$ is available from the third-order nonlinear optical parameters that can be evaluated by fitting the experimental results in the context of the non-linear optical theory. The number of rings $N$ is plotted in Figure $2 \mathbf{F}$ as a function of incident intensity $I$, and the linear relationship between both parameters is evident. According to the Kerr law, the refractive index of $\mathrm{Nb}_{2} \mathrm{C}$ can be obtained as follows [1]:

$$
n=n_{0}+n_{2} I
$$

where $n_{0}=1.47$ (NMP) and $n_{2}$ are the linear and nonlinear refractive indexes, respectively, and $I$ represents the laser beam intensity. When the laser beam passes through the $\mathrm{Nb}_{2} \mathrm{C}$ suspension, the phase shift $(\Delta \Psi)$ can be expressed as follows [1]:

$$
\Delta \psi=\frac{2 \pi n_{0}}{\lambda} \int_{0}^{L_{e f f}} n_{2} I(r, z) d z
$$

where $\lambda$ is the wavelength, $L_{\text {eff }}$ is the effective optical thickness, $r \in[0, \infty)$ is the radial coordinate, and $I(r, z)$ represent the radial intensity distribution of a focused laser beam. The total number 
A

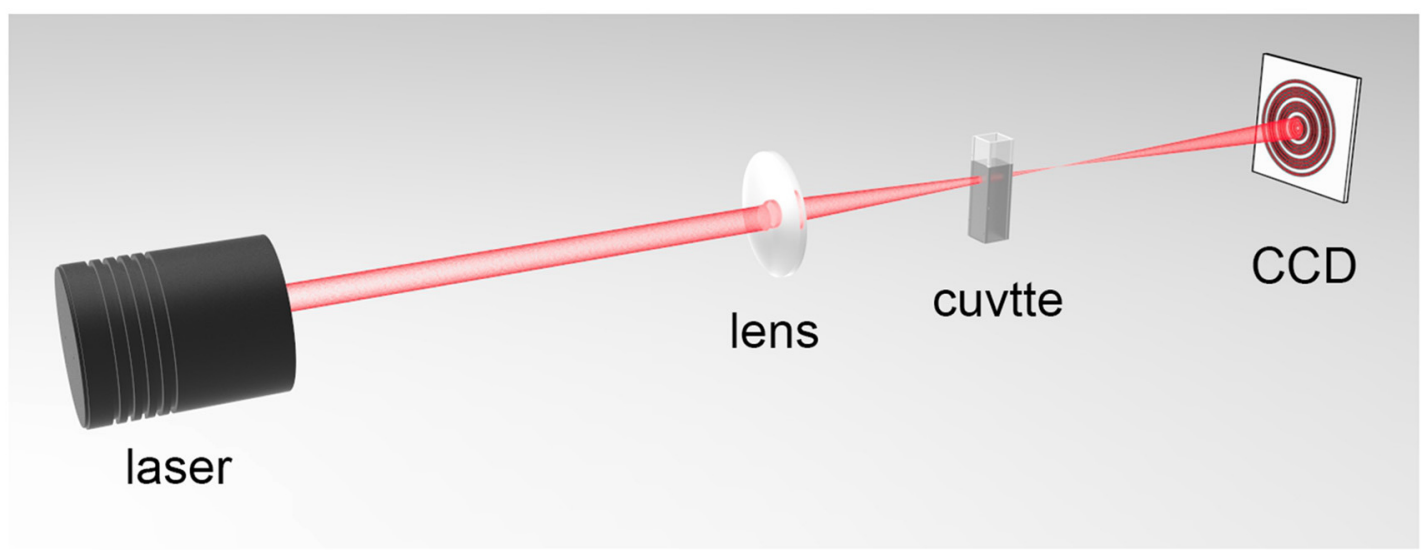

B

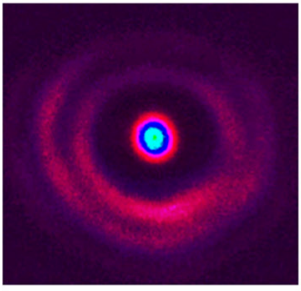

D

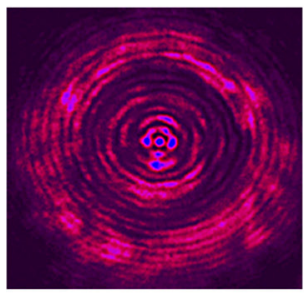

C

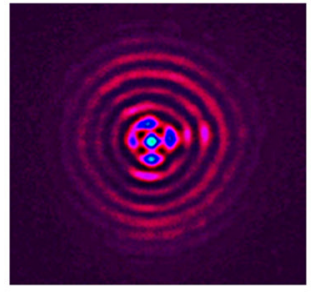

E

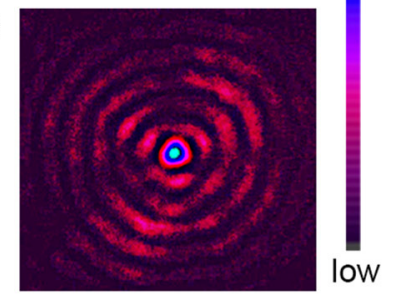

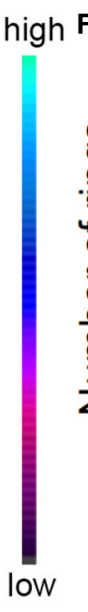

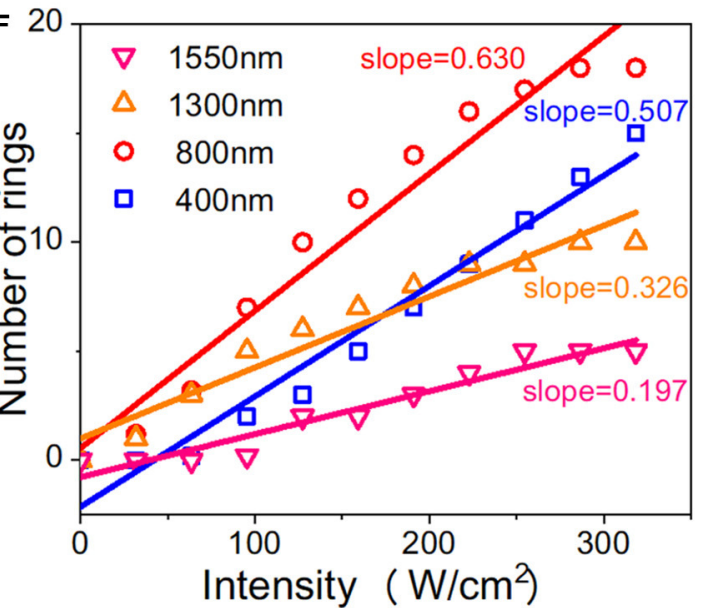

FIGURE 2 | (A) Experimental setup for SSPM measurements. (B-E) SSPM patterns at (B) 1,550 nm, (C) 1,300 nm, (D) $800 \mathrm{~nm}$, (E) $400 \mathrm{~nm}$. (F) Numbers of diffraction rings vs. incident beam intensity at four wavelengths.

of rings $N$ is determined by the formula $\Delta \Psi(0)-\Delta \Psi(\infty)=2 N \pi$ [1]. As for the Gaussian beam, the intensity at the center of the beam $\left(I_{0}\right)$ is twice the average intensity $I$, which can be found experimentally. Then the effective thickness of the cuvette $L_{\text {eff }}$ can be calculated from the following formula [1]:

$$
L_{e f f}=\int_{L_{1}}^{L_{2}}\left(1+\frac{z^{2}}{z_{0}^{2}}\right)^{-1} d z,\left(z_{0}=\frac{\pi \omega_{0}^{2}}{\lambda}\right)
$$

where $L_{1}$ and $L_{2}$ are the distances from the focal point to the back and front surfaces of the quartz cuvette respectively, and $L=L_{2}$ $L_{1}$ is the thickness of the quartz cuvette. In our experiment, the focal length of the lens is $f=20 \mathrm{~cm}, L=1 \mathrm{~cm}$, and $\omega_{0}$ is the waist radius of the laser beam. Therefore, the non-linear index $n_{2}$ can be defined as follows [37]:

$$
n_{2}=\frac{\lambda}{2 n_{0} L_{e f f}} \cdot \frac{N}{I}
$$

To gain insight into the intrinsic non-linear optical property of $\mathrm{Nb}_{2} \mathrm{C}$ nanosheets, the third-order non-linear susceptibility $\chi_{\text {total }}^{(3)}$ can be described by the equation below [37]:

$$
\chi_{\text {total }}^{(3)}=\frac{c \lambda n_{0}}{2.4 \times 10^{4} \pi^{2} L_{e f f}} \cdot \frac{d N}{d I}
$$

Then, $\chi_{\text {monolayer }}^{(3)}$ of $\mathrm{Nb}_{2} \mathrm{C}$ can be obtained as follows [37]:

$$
\chi_{\text {total }}^{(3)}=\chi_{\text {monolayer }}^{(3)} \cdot A_{e f f}^{2}
$$

The number of layers $A_{\text {eff }}$ can be found as [28], where $\rho=$ $0.00286 \mathrm{~mol} / \mathrm{L}$ is the $\mathrm{Nb}_{2} \mathrm{C}$ concentration, and $V=4 \times 10^{-3}$ L. The number of molecules in a single layer $m=1 \times 4$ $\mathrm{cm}^{2} /\left(\sin 60^{\circ}\right) \times(6.302)^{2} \AA^{2}=1.83 \times 10^{16}$. Hence the number of layers is $A_{\text {eff }}=374$.

The experimentally measured non-linear optical parameters of different 2D materials are summarized in Table 1 . The superior non-linear refractive response of $\mathrm{Nb}_{2} \mathrm{C}$ at $1,550 \mathrm{~nm}$ is a unique 
TABLE 1 | Third-Order non-linear optical parameters of different 2D materials.

\begin{tabular}{|c|c|c|c|c|}
\hline 2D materials & Wavelength (nm) & $\mathrm{n}_{2}\left(\mathrm{~cm}^{2} / \mathrm{W}\right)$ & $\chi(3)$ monolayer (e.s.u.) & References \\
\hline $\mathrm{Bi}_{2} \mathrm{Te}_{3}$ & 1,070 & $2.11 \times 10^{-12}$ & $\sim 10^{-9}$ & [31] \\
\hline \multirow[t]{2}{*}{$\mathrm{BP}$} & 700 & $3.10 \times 10^{-5}$ & $3.49 \times 10^{-8}$ & {$[32]$} \\
\hline & 1,160 & $2.81 \times 10^{-5}$ & $3.16 \times 10^{-8}$ & \\
\hline \multirow[t]{2}{*}{$\mathrm{Ti}_{3} \mathrm{C}_{2} \mathrm{~T}_{\mathrm{x}}$} & 800 & $\sim 10^{-18}$ (monolayer) & $\sim 10^{-15}$ & [33] \\
\hline & 1,064 & $\sim 10^{-10}$ (monolayer) & $\sim 10^{-7}$ & \\
\hline \multirow[t]{4}{*}{$\mathrm{Nb}_{2} \mathrm{C}$} & 400 & $6.21 \times 10^{-6}$ & $2.43 \times 10^{-9}$ & Present results \\
\hline & 800 & $1.55 \times 10^{-5}$ & $6.07 \times 10^{-9}$ & \\
\hline & 1,300 & $1.31 \times 10^{-5}$ & $5.14 \times 10^{-9}$ & \\
\hline & 1,550 & $9.53 \times 10^{-6}$ & $3.73 \times 10^{-9}$ & \\
\hline
\end{tabular}

and attractive result. The intrinsic third-order non-linear optical susceptibility of an effective single layer reaches a value of $5.14 \times$ $10^{-9}$ e.s.u. at $1,300 \mathrm{~nm}$ and $3.73 \times 10^{-9}$ e.s.u. at $1,550 \mathrm{~nm}$.

As can be seen in Table $1, \mathrm{Nb}_{2} \mathrm{C}$ possesses relatively high values of non-linear parameters within the near-infrared range. Using femtosecond laser pulses allows one to greatly suppress the thermal effects that may arise during the formation of the diffraction ring patterns due to spatial self-phase modulation $[38,39]$. Non-local electronic coherence is a common mechanism to explain spatial self-phase modulation, which is also known as wind-chime model. However, since the optical bandgap of fewlayer $\mathrm{Nb}_{2} \mathrm{C}$ nanosheets is found to be $0.81 \mathrm{eV}$ [12], the singlephoton energy at $1,550 \mathrm{~nm}$ is not high enough to excite electronic transitions. The parallel-band absorption effects [40] induced two-photon absorption has been discovered in present metallic $\mathrm{Nb}_{2} \mathrm{C}$ nanosheets. Therefore, the two-photon SSPM is the most likely mechanism responsible for the emergence of the below-gap SSPM [28]. When the two-photon absorption establishes a new electric field polarization, the excited carriers will diffract with photons not only above but also below the bandgap [28].

After exploring the formation mechanism of the diffraction ring patterns within the near-infrared wavelength range, we were focused on the investigation of the dynamic formation process of $\mathrm{Nb}_{2} \mathrm{C}$. Figures 3A-D depict the SSPM diffraction patterns acquired using a high-speed CCD detector at different time points and a laser excitation wavelength of $1,300 \mathrm{~nm}$. First, the incidence of the laser beam on a sample leads to its self-focusing (Figure 3A). Then the SSPM-induced diffraction rings appear (Figure 3B). Their number increases rapidly until it becomes maximum. Finally, there is the downward collapse of the upper part of the pattern, forming a perpendicular (asymmetric) stable pattern. Maintaining the same beam incidence conditions, we also obtained the number of rings as a function of the dynamic formation time in Figures 3E-H.

The time required for the diffraction ring formation is found to be almost independent of the incident beam wavelength. A schematic illustration of the time of the rings formation is shown in Figure 4. The first part is the polarization process $\left(t_{0}-t_{1}\right.$ in Figure 4) in which the electrons and holes of the nanosheets move in opposite directions when the laser passes through the sample, causing the nanosheets to be polarized [37]. Such a polarization process can be attributed to the nonlocal electron coherence when there is an interaction between $2 \mathrm{D}$ nanosheets and light-induced electromagnetic field. The second part is the reorientation process $\left(t_{1}-t_{2}\right.$ in Figure 4$)$ in which the polarized fragments are rearranged under the force of an electric field. During the reorientation process, non-local electron coherence among the 2D nanosheets will be formed gradually. Such a non-local electron coherence-induced formation of typical SSPM diffraction rings have been observed in $\mathrm{MoS}_{2}$ system previously [28]. In terms of the response time, the excited carriers generation and 2D nanosheets polarization will appear in the first part which corresponds to a timescale of about a picosecond or even a femtosecond. Considering the second part, there is typical interaction between polarized 2D nanosheets and Newton fluid with constant viscosity coefficient under the laser incidence. This process is a relatively slow one (about millisecond) and will be influenced by many parameters including viscosity coefficient of solvent, size of the 2D nanosheets, and incident laser power [28].

Taking into account the advantage of the SSPM response of $\mathrm{Nb}_{2} \mathrm{C}$, non-reciprocal light propagation was designed as a proof-of-concept nanophotonic device. To construct asymmetric composite film system with non-reciprocal light transmission property, the composite films always contain a saturable absorption layer and a reverse saturable absorption material layer [41]. In the Kerr non-linearity-based, non-reciprocal light transmission work, the energy absorption of the incident light is the most important function of the reverse saturable absorption material layer [27]. We use the water to replace these reverse saturable absorption materials to construct an asymmetric composite film system. As we know, water has the advantages of stable physical and chemical properties and is environmentally friendly. Furthermore, water is low-cost and can be procured everywhere. When the laser beam passes forward $\left(\mathrm{Nb}_{2} \mathrm{C} /\right.$ water $)$, the SSPM diffraction rings will be excited due to a strong SSPM response of $\mathrm{Nb}_{2} \mathrm{C}$ caused by the Kerr effect. In other words, its large non-linear refractive index modulates the phase of the laser beam. Thereafter, water just reduces the intensity of the laser but makes no difference to the number of rings. Figures 5A-D display the SSPM patterns collected at the backward movement of the laser beam through the $\mathrm{Nb}_{2} \mathrm{C} /$ water hybrid structure at different beam intensities and a wavelength of $1,300 \mathrm{~nm}$. However, when the laser passes backward, water reduces the incident light intensity that is small enough not 
A

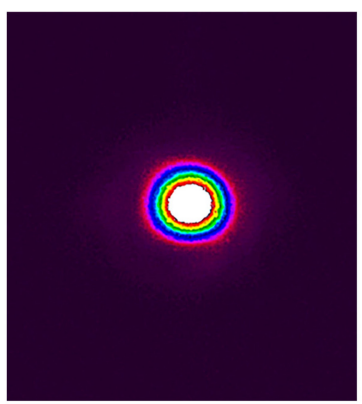

B

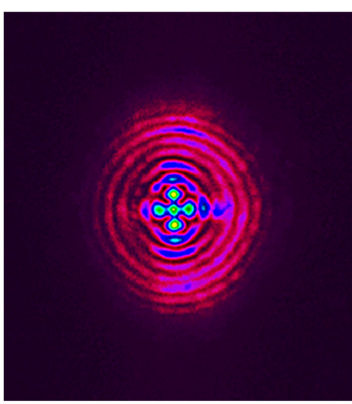

E

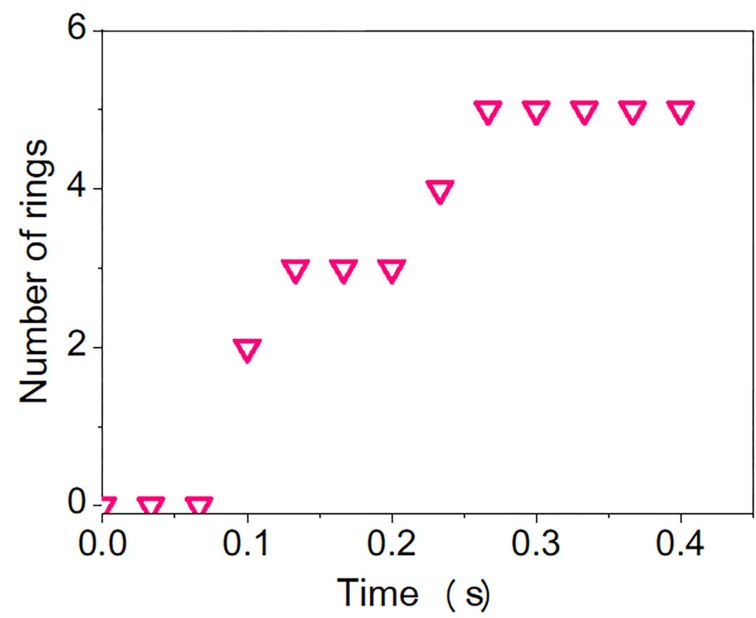

G

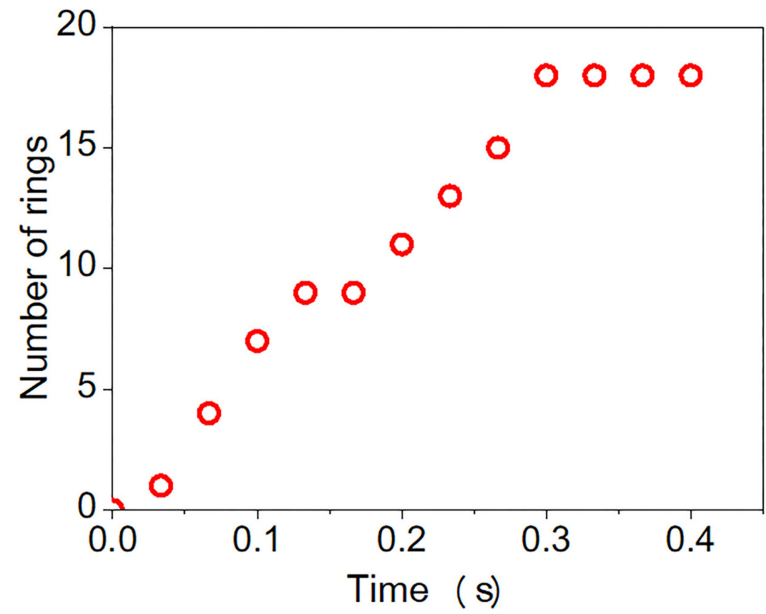

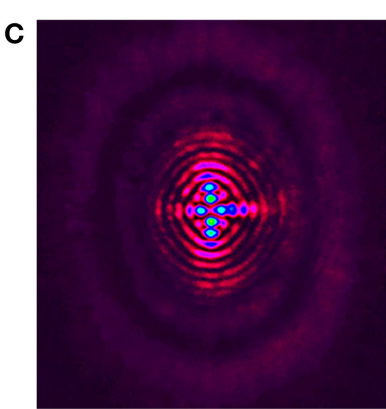

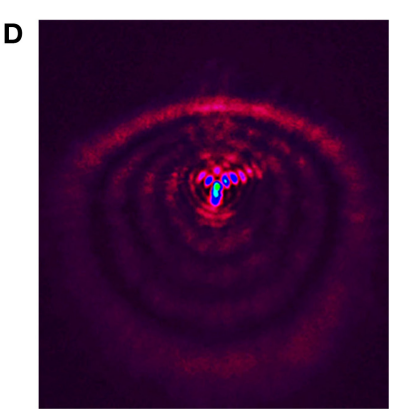

$\mathbf{F}$
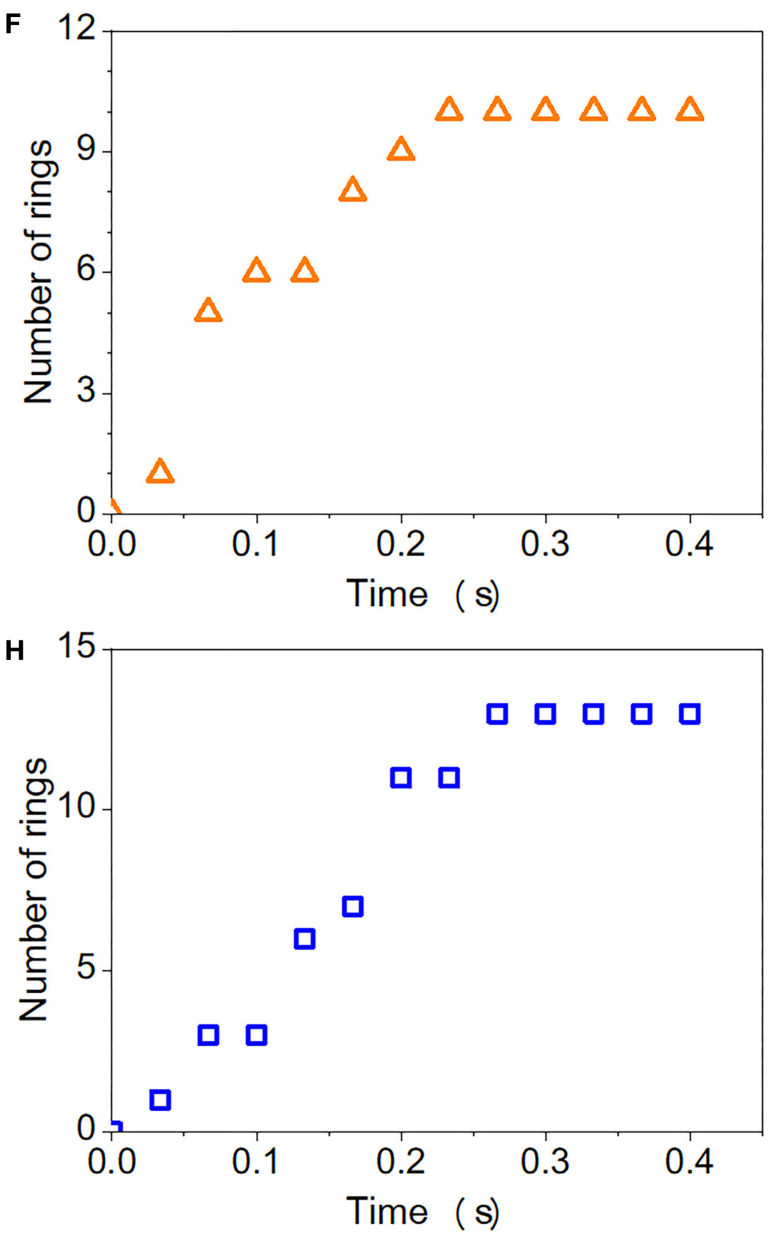

FIGURE 3 | (A-D) Formation process of SSPM patterns at (A) $0 \mathrm{~s}$, (B) $0.07 \mathrm{~s}$, (C) $0.53 \mathrm{~s}$, (D) $1 \mathrm{~s}$ at a wavelength of $1,300 \mathrm{~nm}\left(\right.$ laser beam intensity I = $\left.31.8 \mathrm{~W} / \mathrm{cm}^{2}\right)$. (E-H) Number of rings $N$ changing with the time at a wavelength of (E) $1,550 \mathrm{~nm}$, (F) 1,300 nm, (G) $800 \mathrm{~nm}$, (H) $400 \mathrm{~nm}$, respectively.

to excite diffraction rings in the $\mathrm{Nb}_{2} \mathrm{C}$ layer due to its strong absorption in the near-infrared range. Figures 5E,F summarize the results of non-reciprocal light propagation for $\mathrm{a} \mathrm{Nb}_{2} \mathrm{C} /$ water hybrid structure at wavelengths of 1,300 and $1,550 \mathrm{~nm}$, where it easily observes the unidirectional excitation of the diffraction rings. This discovery not only enabled one to find a new medium for the non-reciprocal light propagation implementation, but also to broaden the insight into the non-linear optical devices operating within the near-infrared range (especially at $1,550 \mathrm{~nm})$.

\section{CONCLUSION}

In summary, few-layer $\mathrm{Nb}_{2} \mathrm{C}$ nanosheets have been synthesized and their non-linear optical characteristics were measured via the SSPM technique within a near-infrared broadband range. The few-layer $\mathrm{Nb}_{2} \mathrm{C}$ nanosheets were shown to exhibit excellent nonlinear optical response and their non-linear refractive index was as high as $9.53 \times 10-6 \mathrm{~cm}^{2} / \mathrm{W}$ at $1,550 \mathrm{~nm}$. Moreover, in order to understand the physics mechanism behind it, dynamic formation process of SSPM diffraction rings was exploited. Based on the 
polarized nanosheets unpolarized nanosheets
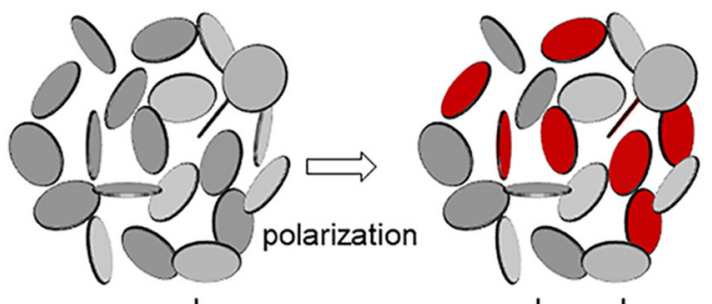

rentation
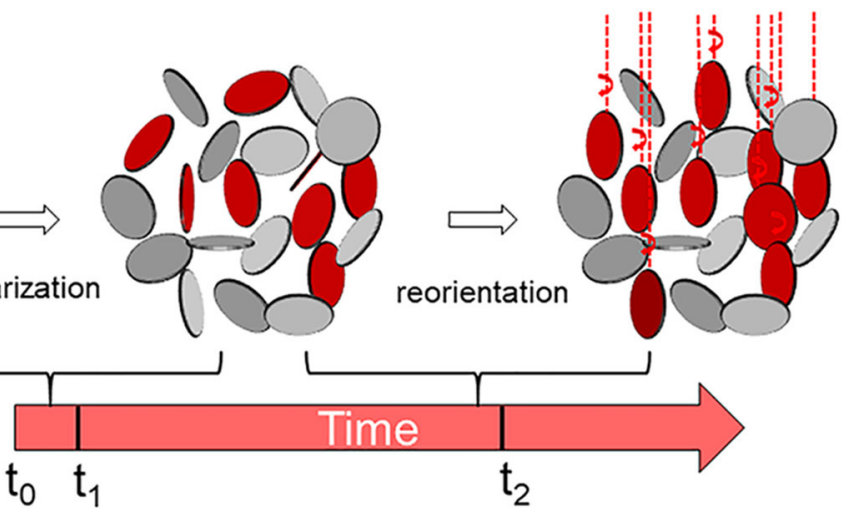

Time

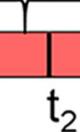

FIGURE 4 | Schematic illustration of the time required for the diffraction ring formation. The time can be divided into two parts. The $t_{0}$ indicates the laser start to incident on the medium. The polarization of $2 \mathrm{D}$ nanosheets finished in $\mathrm{t}_{0}-\mathrm{t}_{1}$ region. The reorientation of $2 \mathrm{D} \mathrm{Nb_{2 }} \mathrm{C}_{\text {occurs in }} \mathrm{t}_{1}-\mathrm{t}_{2}$ time zone. $\mathrm{t}_{0}-\mathrm{t}_{2}$ time zone represents the time of the diffraction ring formation. $t_{2}$ diffraction rings become maximum.

A

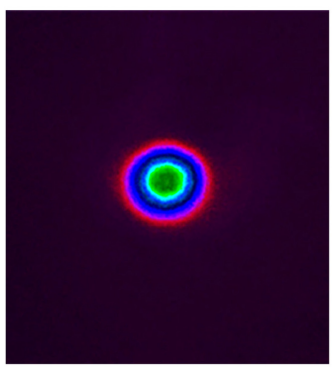

B

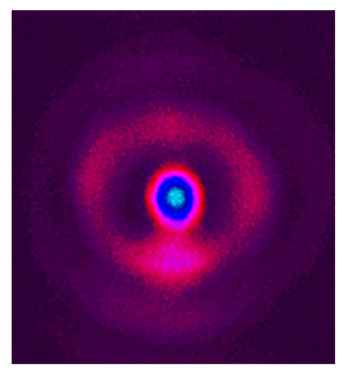

c

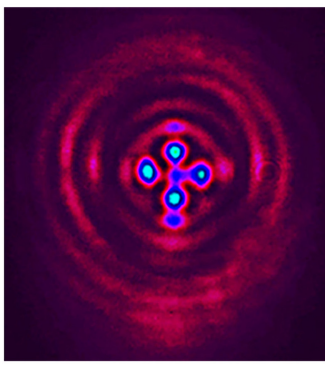

D

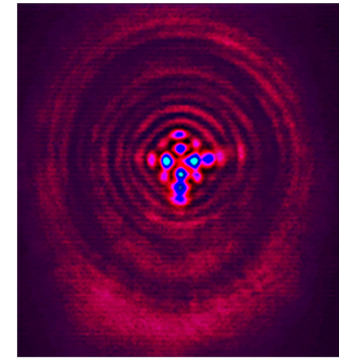

E

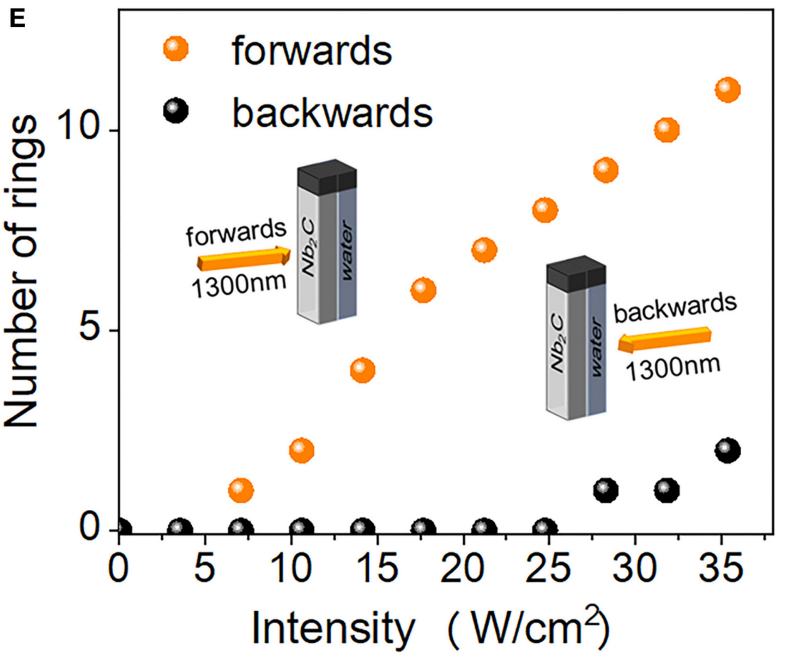

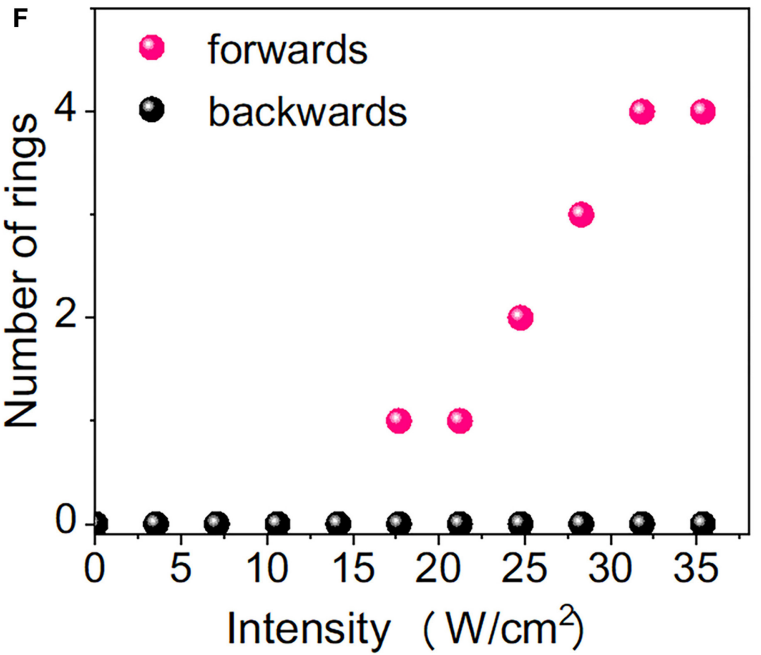

FIGURE 5 | (A-D) SSPM patterns (acquired at the forward advancement of the laser beam) of a $\mathrm{Nb}_{2} \mathrm{C} /$ water hybrid structure at a wavelength of $1,300 \mathrm{~nm}$ and laser beam intensities of (A) $3.5 \mathrm{~W} / \mathrm{cm}^{2}$, (B) $10.6 \mathrm{~W} / \mathrm{cm}^{2}$, (C) $21.2 \mathrm{~W} / \mathrm{cm}^{2}$, (D) $31.8 \mathrm{~W} / \mathrm{cm}^{2}$. (E,F) Number of diffraction rings vs. laser beam intensity at (E) $1,300 \mathrm{~nm}$ and (F) $1,550 \mathrm{~nm}$ at the forward (backward) movement of a laser beam through the hybrid structure. The inserted images explain the beam incidence direction. 
time of the rings formation, the dynamic formation process of SSPM diffraction rings can be divided into two typical time parts which correspond to the polarization and reorientation of $2 \mathrm{D}$ $\mathrm{Nb}_{2} \mathrm{C}$ nanosheets. Finally, the non-reciprocal light propagation was implemented through a $\mathrm{Nb}_{2} \mathrm{C}$ /water hybrid structure, giving a strong boost to research in the field of non-linear nanophotonic applications.

\section{DATA AVAILABILITY STATEMENT}

The original contributions presented in the study are included in the article/supplementary material, further inquiries can be directed to the corresponding author/s.

\section{REFERENCES}

1. Durbin SD, Arakelian SM, Shen YR. Laser-induced diffraction rings from a nematic-liquid-crystal film. Opt Lett. (1981) 6:411-3. doi: 10.1364/OL.6.000411

2. Wu L, Yuan X, Ma D, Zhang Y, Huang W, Ge Y, et al. Recent advances of spatial self-phase modulation in $2 \mathrm{D}$ materials and passive photonic device applications. Small. (2020) 16:2002252. doi: 10.1002/smll.202002252

3. Yu S, Wu X, Wang Y, Guo X, Tong L. 2D materials for optical modulation: challenges and opportunities. Adv Mater. (2017) 29:1606128. doi: 10.1002/adma.201606128

4. Sun Z, Martinez A, Wang F. Optical modulators with 2D layered materials. Nat Photonics. (2016) 10:227-38. doi: 10.1038/nphoton.2016.15

5. Bao Q, Zhang H, Wang Y, Ni Z, Yan Y, Shen ZX, et al. Atomic-layer graphene as a saturable absorber for ultrafast pulsed lasers. Adv Func Mater. (2009) 19:3077-83. doi: 10.1002/adfm.200901007

6. Wang Y, Mu H, Li X, Yuan J, Chen J, Xiao S, et al. Observation of large nonlinear responses in a graphene-Bi2 $\mathrm{Te} 3$ heterostructure at a telecommunication wavelength. Appl Phys Lett. (2016) 108:221901. doi: 10.1063/1.4953072

7. Wang K, Wang J, Fan J, Lotya M, O’Neill A, Fox D, et al. Ultrafast saturable absorption of two-dimensional MoS2 nanosheets. ACS Nano. (2013) 7:92607. doi: $10.1021 / \mathrm{nn} 403886 \mathrm{t}$

8. Wang K, Szydłowska BM, Wang G, Zhang X, Wang JJ, Magan JJ, et al. Ultrafast nonlinear excitation dynamics of black phosphorus nanosheets from visible to mid-infrared. ACS Nano. (2016) 10:6923-32. doi: 10.1021/acsnano.6b02770

9. Wang Y, Huang G, Mu H, Lin S, Chen J, Xiao S, et al. Ultrafast recovery time and broadband saturable absorption properties of black phosphorus suspension. Appl Phys Lett. (2015) 107:091905. doi: 10.1063/1.4930077

10. Wang Y, Liu S, Zeng B, Huang H, Xiao J, Li J, et al. Ultraviolet saturable absorption and ultrafast carrier dynamics in ultrasmall black phosphorus quantum dots. Nanoscale. (2017) 9:4683-90. doi: 10.1039/C6NR09235G

11. Wang G, Bennett D, Zhang C, Ó Coileáin C, Liang M, McEvoy N, Wang JJ, et al. Two-photon absorption in monolayer Mxenes. Adv Opt Mater. (2020) 8:1902021. doi: 10.1002/adom.201902021

12. Wang Y, Wang Y, Chen K, Qi K, Xue T, Zhang H, et al. NiobiumCarbide mxenes with broadband nonlinear optic response and ultrafast carrier dynamics. ACS Nano. (2020) 14:10492-10502. doi: 10.1021/acsnano.0c04390

13. Wu Y, Zhu LL, Wu Q, Sun F, Wei JK, Tian Y, et al. Electronic origin of spatial self-phase modulation: evidenced by comparing graphite with C60 and graphene. Appl Phys Lett. (2016) 108:241110. doi: 10.1063/1.4953827

14. He L, Ye J, Shuai M, Zhu Z, Zhou X, Wang Y, et al. Graphene oxide liquid crystals for reflective displays without polarizing optics. Nanoscale. (2015) 7:1616-22. doi: 10.1039/C4NR06008C

15. Xiao S, Ma Y, He Y, Wang Y, Xin H, Fan Q, et al. Revealing the intrinsic nonlinear optical response of a single MoS 2 nanosheet in a suspension based on spatial self-phase modulation. Photonics Res. (2020) 8:172533. doi: 10.1364/PRJ.399364

\section{AUTHOR CONTRIBUTIONS}

SX and JH conceived the idea. Y-wW, Y-lD, Y-dW, and LZ prepared the $2 \mathrm{D}$ material and conducted the characterization of $2 \mathrm{D}$ material. $\mathrm{Y}-\mathrm{lH}, \mathrm{X}-\mathrm{jZ}$, and $\mathrm{SX}$ performed SSPM measurement of 2D material and analyzed results. All authors involved in the discussion and manuscript writing.

\section{FUNDING}

This work was financially supported by the National Natural Science Foundation of China (61875232, 61874141, and 11904239).

16. Hu L, Sun F, Zhao H, Zhao J. Nonlinear optical response spatial self-hase modulation in MoTe2: correlations between X(3) and mobility or effective mass. Opt Lett. (2019) 44:5214-17. doi: 10.1364/OL.44.005214

17. Wu L, Huang W, Wang Y, Zhao J, Ma D, Xiang Y, et al. 2D Tellurium Based High-Performance all-optical nonlinear photonic devices. Adv Func Mater. (2019) 29:1806346. doi: 10.1002/adfm.201806346

18. Jia Y, Liao Y, Wu L, Shan Y, Dai X, Cai H, et al. Nonlinear optical response, all optical switching, and all optical information conversion in NbSe2 nanosheets based on spatial self-phase modulation. Nanoscale. (2019) 11:4515-22. doi: 10.1039/C8NR08966C

19. Shan Y, Wu L, Liao Y, Tang J, Dai X, Xiang Y. A promising nonlinear optical material and its applications for all-optical switching and information converters based on the spatial self-phase modulation (SSPM) effect of TaSe2 nanosheets. J Mater Chem C. (2019) 7:3811-6. doi: 10.1039/C9TC00333A

20. Jia Y, Li Z, Saeed M, Tang J, Cai H, Xiang Y. Kerr nonlinearity in germanium selenide nanoflakes measured by Z-scan and spatial self-phase modulation techniques and its applications in all-optical information conversion. Opt Express. (2019) 27:20857-73. doi: 10.1364/OE.27.020857

21. Wang W, Wu Y, Wu Q, Hua J, Zhao J. Coherent nonlinear optical response spatial self-phase modulation in MoSe2 nano-sheets. Sci Rep. (2016) 6:22072. doi: 10.1038/srep22072

22. Li X, Liu R, Xie H, Zhang Y, Lyu B, Wang P, et al. Tri-phase alloptical switching and broadband nonlinear optical response in Bi2Se3 nanosheets. Opt Express. (2017) 25:18346-54. doi: 10.1364/OE.25. 018346

23. Biswas S, Kumbhakar P. Measurement of large nonlinear refractive index of natural pigment extracted from hibiscus rosa-sinensis leaves with a low power CW laser and by spatial self-phase modulation technique. Spectrochim Acta A. (2017) 173:400-6. doi: 10.1016/j.saa.2016.09.049

24. Jia Y, Shan Y, Wu L, Dai X, Fan D, Xiang Y. Broadband nonlinear optical resonance and all-optical switching of liquid phase exfoliated tungsten diselenide. Photonics Res. (2018) 6:1040-7. doi: 10.1364/PRJ.6.001040

25. Wang G, Higgins S, Wang K, Bennett D, Milosavljevic N, Magan JJ, et al. Intensity-dependent nonlinear refraction of antimonene dispersions in the visible and near-infrared region. Appl Optics. (2018) 57:E14753. doi: 10.1364/AO.57.00E147

26. Ji W, Chen W, Lim S, Lin J, Guo Z. Gravitation-dependent, thermallyinduced self-diffraction in carbon nanotube solutions. Opt Express. (2006) 14:8958-66. doi: 10.1364/OE.14.008958

27. Wu L, Dong Y, Zhao J, Ma D, Huang W, Zhang Y, et al. Kerr nonlinearity in 2D graphdiyne for passive photonic diodes. Adv Mater. (2019) 31:1807981. doi: 10.1002/adma.201807981

28. Wu Y, Wu Q, Sun F, Cheng C, Meng S, Zhao J. Emergence of electron coherence and two-color all-optical switching in MoS2 based on spatial self-phase modulation. Proc Natl Acad Sci USA. (2015) 112:118005. doi: 10.1073/pnas.1504920112

29. Wang Y, Tang Y, Cheng P, Zhou X, Zhu Z, Liu Z, et al. Distinguishing thermal lens effect from electronic third-order nonlinear self-phase modulation 
in liquid suspensions of 2D nanomaterials. Nanoscale. (2017) 9:354754. doi: 10.1039/C6NR08487G

30. Xiao S, Zhang Y, Ma Y, Wang Y, He Y, Zhang J, et al. Observation of spatial selfphase modulation induced via two competing mechanisms. Opt Lett. (2020) 45:2850-3. doi: 10.1364/OL.392689

31. Shi B, Miao L, Wang Q, Du J, Tang P, Liu J, et al. Broadband ultrafast spatial self-phase modulation for topological insulator Bi2Te3 dispersions. Appl Phys Lett. (2015) 107:151101. doi: 10.1063/1.4932590

32. Zhang J, Yu X, Han W, Lv B, Li X, Xiao S, et al. Broadband spatial self-phase modulation of black phosphorous. Opt Lett. (2016) 41:17047. doi: 10.1364/OL.41.001704

33. Li J, Zhang Z, Yi J, Miao L, Huang J, Zhang J, et al. Broadband spatial selfphase modulation and ultrafast response of MXene Ti3C2Tx $(\mathrm{T}=\mathrm{O}, \mathrm{OH}$ or F). Nanophotonics. (2020) 9:2415-24. doi: 10.1515/nanoph-2019-0469

34. Du L, Duan H, Xia Q, Jiang C, Yan Y, Wu S. Hybrid charge-storage route to Nb2CTx MXene as anode for sodium-ion batteries. ChemistrySelect. (2020) 5:1186-92. doi: 10.1002/slct.201903888

35. Pang SY, lo WF, Wong LW, Zhao J, Hao J. Efficient energy conversion and storage based on robust fluoride-free self-assembled 1D niobium carbide in 3D nanowire network. Adv Sci. (2020) 7:1903680. doi: 10.1002/advs.201903680

36. Lin H, Gao S, Dai C, Chen Y, Shi J. Two-Dimensional biodegradable niobium carbide (MXene) for photothermal tumor eradication in NIR-I and NIR-II bio-windows. J Am Chem Soc. (2017) 139:16235-47. doi: 10.1021/jacs.7b07818

37. Wu R, Zhang Y, Yan S, Bian F, Wang W, Bai X, et al. Purely coherent nonlinear optical response in solution dispersions of graphene sheets. Nano Lett. (2011) 11:5159-64. doi: 10.1021/nl2023405
38. Qi P, Su Q, Lu D, Lin L, Zhang N, Liu W. Optical nonlinearities of alcoholic liquids under high-repetition-rate femtosecond lasers by single beam time-resolved eclipsed Z-scan. Opt Laser Technol. (2019) 109:643-7. doi: 10.1016/j.optlastec.2018. 08.054

39. Falconieri M, Salvetti G. Simultaneous measurement of pure-optical and thermo-optical nonlinearities induced by high-repetition-rate, femtosecond laser pulses: application to CS2. Appl Phys B. (1999) 69:133-6. doi: 10.1007/s003400050785

40. Harrison WA. Parallel-band effects in interband optical absorption. Phys Rev. (1966) 147:467. doi: 10.1103/PhysRev.147. 467

41. Anand B, Podila R, Lingam K, Krishnan SS, Sai SS, Philip R, et al. Optical diode action from axially asymmetric nonlinearity in an all-carbon solid-state device. Nano Lett. (2013) 13:5771-6. doi: 10.1021/nl403366d

Conflict of Interest: The authors declare that the research was conducted in the absence of any commercial or financial relationships that could be construed as a potential conflict of interest.

Copyright (c) 2021 Xiao, He, Dong, Wang, Zhou, Zhang, Wang and He. This is an open-access article distributed under the terms of the Creative Commons Attribution License (CC BY). The use, distribution or reproduction in other forums is permitted, provided the original author(s) and the copyright owner(s) are credited and that the original publication in this journal is cited, in accordance with accepted academic practice. No use, distribution or reproduction is permitted which does not comply with these terms. 